\title{
COMING EVENTS
}

- Signifies events wholly or partially sponsored by IUGS, one of its Affiliated Associations or joint ventures - the International Geological Correlation Programme (IGCP) or the Inter-Union Commission on the Lithosphere (ICL).

- Signifies first announcement of meeting in EPISODES.

\section{4}

May 2, 1984

- SOURCES of GEOLOGICAL INFORMATION FOR THE FAR EAST (Geological Society Meeting) London. (J. Hopson, Aldwych Reading Room, Science Reference Library, Kean Street, London WC2B 4AT, U.K.)

May $6-11,1984$

CASE HISTORIES IN GEOTECHNICAL ENGINEERING (International Conference), Rolla, Missouri, U.S.A. (W. Kratzer, Engineering Continuing Education, 111 ERL, University of Missouri-Rolla, Rolla, MO 65401, U.S.A.)

May $6-8,1984$

- RECENT ADVANCES IN THE GEOCHEMISTRY OF ORE DEPOSITS (Conference), Montreal, Canada. (Valérje Virard, Mineral Exploration Research Institute, C.P. 6079, Succursale A, Montréal, Québec, Canada H3C 3A7)

May $7-11,1984$

- EFFECTS OF FOREST LAND USE ON EROSION AND SLOPE STABILITY (Symposium)' Honolulu, Hawaii, U.S.A. (IUFRO Symposium, c/o Redwood Sciences Laboratory, 1700 Bayview Drive, Arcata, CA 95521, U.S.A.)

May $8-10,1984$

MINERALOGIE DANS LES SCIENCES DE LA TERRE ET L'INDUSTRIE (Symposium), Toulouse, France. (F. Fontan, Laboratoire de Minéralogie et de Cristallographie, 39, allées Jules-Guesde, 31400 Toulouse, France)

May $10-13,1984$

MINERALIZATION IN VOLCANIC PROCESSES (Geological Society Meeting). Aberystwyth, Wales, U.K. (Geological Society, Burlington House, Piccadilly, London W IV OJU, U.K.)

Mav $14-16,1984$

GEOLOGICAL ASSOCIATION OF CANADA, MINERALOGICAL ASSOCIATION OF CANADA (Joint Annual Meeting), London, Ontario, Canada. (N.D. MacRae, Department of Geology, University of Western Ontario, London, Ont., Canada N6A 5B7)

Mav $14-17,1984$

DRAINAGE BASIN EROSION AND SEDIMEN. TATION (Conference), University of Newcastle, V.S.W. Australia. Sponsored by IAHS. (Dr. R.J. Loughran, Department of Geography, University of Newcastle, N.S.W. 2308, Alis tralia)

May $14-18,1984$

AMERICAN GEOPHYSICAL UNION (Spring Meeting), Cincinnati, Ohio, U.S.A. (Meetings, AGU, 2000 Florida Avenue NW, Washington, DC 20009, U.S.A.)

May $14-18,1984$

GEOMECHANICS (4th Australia-New Zealand Conference), Perth, Western Australia. (The Conference Manager, 4th A.N.Z. Geomechanics Conference, The Institution of Engineers, Australia, 11 National Circuit, Barton, ACT 2600, Australia)

May $15-19,1984$

THRUSTING AND DEFORMATION (International Conference), Toulouse, France. Field trips. Languages: English and French. ( $J$. Deramond, Laboratoire de géologie-pétrologie, Université Paul Sabatier, 38 rue des 36 Ponts, 31078 Toulouse Cédex, France)

May $17-18,1984$

PROSPECTING IN AREAS OF GLACIATED TERRAIN, Glasgow, Scotland. (Conference Office, IMM, 44 Portland Place, London WIN 4BR, U.K.)
May $20-23,1984$

AAPG and SEPM (Joint Annual Meeting), San Antonio, Texas, U.S.A. (AAPG, Box 979, Tulsa, OK 74101 , U.S.A.)

May 20 - 23, 1984

INDUSTRIAL MINERALS (6th International Congress), Toronto, Canada. (B.M. Coope - Editor, Industrial Minerals, 45/46 Lower Marsh, London SEI, U.K.)

May $20-25,1984$

- OBSERVATION OF THE CONTINENTAL CRUST THROUGH DRILLING (International Symposium) Tarrytown, N.Y., U.S.A. Co-sponsored by ICL. (Margaret Swan, Lamont-Doherty Geological Observatory, Palisades, NY 10964, U.S.A.)

May $21-23,1984$

AGGREGATES (International Symposium), Nice, France. Sponsored by IAEG. Languages: English and French. (M. Louis Primel, LCPC, 58 boulevard Lefébure, 75732 Paris Cedex 15, France)

May $21-23,1984$

CLIMATE: PRESENT, PAST, AND FUTURE (Symposium), New York, N.Y., U.S.A. Sponsored by Columbia University in honour of Rhodes W. Fairbridge. (John E. Sanders, Department of Geology, Columbia University, 606 West 120th Street, New York, NY 10027, U.S.A.)

May $21-23,1984$

- GROUNDWATER RESOURCE UTILIZATION AND CONTAMINANT HYDROGEOLOGY (International Symposium), Montreal, Quebec, Canada. Sponsored by Canadian National Chapter of IAH and CWWA. Languages: English and French. (Mr. H. Sommelet, Geomines Ltd., 1010 Sherbrooke St. W., Suite 2202, Montreal, Quebec, Canada H3A 2R7)

May 28 - June 2, 1984

IRRIGATION AND DRAINAGE (International Congress), Fort Collins, Colorado, U.S.A. (U.S. Committee on Irrigation, Drainage \& Flood Control, P.O. Box 15326, Denver, CO 80215 , U.S.A.)

May 30 - June 3,1984

- THE RHENISH MASSIF (International WorkshopMeeting), Boppard/Rhein, Germany, Language English. (Dr. Reinhard Greiling, Inst. für Geowissenschaften, Johannes Gutenberg Universität, Saarstrasse 21, D-6500 Mainz 1, F.R.G)

June $3-7,1984$

- TUNNELliNG 84 (Symposium) Caracas, Venezuela. (Secretario General, ITA Tuneles 1984 Apdo 68265, Caracas 1062 A, Venezuela)

June $4-6,1984$

MATHEMATICAL MODELLING OF SEDIMENT TRANSPORT AND MORPHOLOGY (Euromech Colloguium), The Netherlands. (Prof. H.H. Fernholz. Hermann-Föttinger-Institut für ThermoFluiddynamik, Technische Universität Berlin, Strasse des 17 Juni 135, D-1000 Berlin 12, F.R.G.)

June $4-6,1984$

- PALAEOCLIMATOLOGY OF LAKES, RIVERS AND GLACIERS (International Symposium), Innsbruck, Austria. Co-sponsored by ICCL and INQUA. (Prof. M. Kuhn, Institute for Meteorology and Geophysics, Schopfstrasse 41, A-6020 Innsbruck, Austria)

June $4-7,1984$

SOIL MECHANICS AND FOUNDATION ENGINEERING (8th African Regional Conference) Harare, Zimbabwe. (ARC-8, P.O. Box MP 155 , Mount Pleasant, Harare, Zimbabwe)
June $4-8,1984$

DEEP SEA ClASTIC SEdIMENTATION (Short course and Field Trips) French Maritime Alps. (Ph. Riché ENSPM, Institut Français du Pétrole, 4 Ave, de Bois-Preau, 92506-Rueil Malmaison, France)

June 4-10, 1984

READING PROVENANCE FROM ARENITES (NATO Institute), Rende (Cosenza), Italy. (G.G. Zuffa, Dip. Scienze della Terra, Univ. Calabria, 87030 Castiglione Cosentino, Stazione Cosenza, Italy)

June $5+11,1984$

GEOLOGY OF THE HIMALAYAS (International) Symposium), Chengdu City, Sichuan Province, P.R. China. Languages: Chinese, English, French. Excursions. (Li Tingdong, SecretaryGeneral, Organizing Committee, International Symposium on Geology of the Himalayas, c/o Chinese Academy of Geological Sciences, Baiwanzhuang Road 26, Fuchengmenwai, Beijing, People's Republic of China)

June $6-9,1984$

COORDINATION IN FLOOD CONTROL (INTERPRAEVENT 1984), Villach, Austria. (Interpraevent 1984, Postfach 134, A-9501, Villach, Austria)

June $10-14,1984$

PRECIOUS METAL RECOVERY (International Symposium), Reno, Nevada, U.S.A. Post-symposium tour. (International Symposia for Mining, 500 Howard Street, San Francisco, CA 94105 , U.S.A.)

June $11-12,1984$

ENVIRONMENTAL POLLUTION (5th European Conference), Amsterdam, The Netherlands. (Dr. V.M. Bhatnager, Box 1779, Cornwall, Ontario, Canada $\mathrm{K} 6 \mathrm{H} 5 \mathrm{~V} 7 \mathrm{7}$

June $15-17,1984$

SEDIMENTOLOGY OF SHELF SANDS AND SANDSTONES (Research Symposium), Calgary, Canada. (R. John Knight, Petro-Canada, P.O. Box 2844, Calgary, Alberta, Canada T2P 3E 3)

June $18-23,1984$

7th INTERNATIONAL PEAT CONGRESS, Dublin, Ireland. Study tours and post-congress tours. (International Peat Congress, clo Bord na Mona, Lower Baggot Street, Dublin 2, Ireland)

June $19-22,1984$

EUROPEAN ASSOCIATION OF EXPLORATION GEOPHYSICISTS (46th Meeting), London. (Conference Co-Ordinates, 70 Richmond Road, Twickenham, Middlesex, TW 1 3BE, U.K.)

June $20-23,1984$

GEOMEMBRANES (International Conference), Denver, Colorado, U.S.A. Precedes the Impermeable Barriers for Soil and Rock Symposium. (A. Ivan Johnson, Woodward-Clyde Consultants, P.O. Box 4036, Denver, CO 20204, U.S.A.)

Tune $23-26,1984$ PRACTICAL APPLICATIONS OF GROUNDWATER GEOCHEMISTRY (Workshop), Banff Alberta, Canada. (Dr. E.I. Wallick, Alberta Research Council, 5th Floor Terrace Plaza, 4445 Calgary Trail South, Edmonton, Alberta, Canada T6H 5R7)

June $23-30,1984$

MELANGES OF THE APPALACHIAN OROGEN (Penrose Conference), Stephenville, Newfoundland. $(H$. Williams, Department of Earth Sciences, Memorial University, St. Johns, Newfoundland, Canada A1B 3×5) 


\section{Coming Events}

June 24,1984

IMPERMEABLE BARRIERS FOR SOIL AND ROCK (International Symposium), Denver, Colorado, U.S.A. (P.B. Whiteaker, American Society for Testing and Materials, 1916 Race Street, Philadelphia, PA 19103, U.S.A.)

June $24-27,1984$

CODATA ( 9 th International Conference), Jerusalem, Israel. (The Secretariat, 9th International CODATA Conference, 122 Hayarkon Street, P.O. Box 3054,61030 Tel Aviv, Israel)

June 24 - 30, 1984

MATHEMATICAL GEOPHYSICS (14th International Conference), Loen, Norway. (Dr. Durk Doornbos, NTNF/NORSAR, P.O. Box 51, N-2007 Kjeller, Norway; or Dr. Frank Richter, University of Chicago, Chicago, IL 60637, U.S.A.)

June 25 - 29, 1984

- THE CHEMISTRY OF WEATHERING (NATO Workshop), Bonas, France. (J.I. Drever, Geology Dept., Univ. of Wyoming, Laramie, WY 82071 , U.S.A.)

June 25 - July 7,1984

- Cospar (25th Meeting), Graz, Austria. Symposia and Workshops co-sponsored by IUGS include: Planetology of Venus, Mars, and satellites of Outer Planets; and satellite remote sensing of interest to developing countries. Also includes symposium on space observations for climate studies. (COSPAR Secretariat, 51 Boulevard de Montmorency, 75016 Paris, France)

June $26-28,1984$

DEEP STRUCTURE OF THE CONTINENTAL CRUST (International Symposium), Ithaca, N.Y. U.S.A. (Muawia Barazangi, Department of Geological Sciences, Cornell University, Ithaca, NY 14853, U.S.A.)

June $27-29,1984$

- CANNING BASIN (Symposium), Perth, Western Australia. (Phil Connard, Shell Development Pty. Ltd., G.P.O. Box W2050, Perth WA 6001, Australia)

July 1984

VOLCANIC SOILS (International Panel), Tenerife, Canary Islands. (M.E. Fernandez Caldas, Dpto. de Edafologia, Univ, de la Laguna, Tenerife, Islas Canarias, Spain)

July 1984

EROSION AND DESERTIFICATION IN AFRICA (International Workshop), Nigeria. (E.O. Oladipo, Dept. Geography, Ahmadu Bello University, Zarie, Nigeria)

July 1984

RADIOLARISTES (4th Meeting, Eurorad), Leningrad, U.S.S.R. (P. de Wever, Université des Sciences de Lille, Eurorad, U.E.R. Sciences de la Terre, 59655 Villeneuve - d'Ascq, Cedex, France)

July $3-5,1984$

- a pALEOHYDRologiCAL CHANGES IN THE TEMPERATE ZONE IN THE LAST 15000 YEARS (Annual Meeting IGCP 158b), Marseille, France. Co-convened by Euro-Siberian Sub-commission of INQUA-Holocene Commission. (Dr. J.-L. de Beaulieu, Université des Sciences et Techiques de Marseille St. Jérôme, Labo de Botanique, F-13397 Marseille Cedex 13, France)

July $9-12,1984$ GROUND MOVEMENTS AND STRUCTURES (3ro International Conference), Cardiff, Wales, U.K. (The Conference Secretary, Department of Civil Engineering and Building Technology, UWIST, Colum Drive, Cardiff CFI 3EU, South Glamorgan, Wales, U.K.)

July $9-13,1984$

- SPACE TECTONICS FOR GEODYNAMICS (International Symposium), Sopron, Hungary. Cosponsored in part by IAG. (Ch. Reigber, Deutsches Geodätisches For schungsinstitut, Abt. I, Marstallplatz 8, D-8000 Munich 22, F.R.G.)
July $15-18,1984$

FOSSIL FUELS OF EUROPE (AAPG International Conference), Geneva, Switzerland. (AAPG Convention Department, P.O. Box 979, Tulsa, OK 74101 , U.S.A.)

July $16-30,1984$

THE DEEP PROTEROZOIC CRUST IN THE NORTH ATLANTIC PROVINCES (NATO Institute), Moi, Norway. (J. Touret, Inst. Aardwetenschappen, Vrije Univ., Postbus 7161, 1007 MC Amsterdam, Holland)

July $16-18,1984$ MIDDLE TO LATE PROTEROZOIC LITHOSPHERE EVOLUTION (International Conference), Cape Town, South Africa. (Secretary, Precambrian Research Unit, University of Cape Town, Rondebosch 7700 , South Africa)

July $21-28,1984$

EARTHQUAKE ENGINEERING (8th World Conference), San Francisco, Calif., U.S.A. (R.B. Matthisen, Chairman - 8WCEE, Earthquake Engineering Research Institute, 2620 Telegraph Ave., Berkeley, CA 94704 , U.S.A.)

July $23-26,1984$

URBAN HYDROLOGY ( 1 ith International Symposium), Lexington, Kentucky, U.S.A. (E. Haden, Office of Continuing Education/Engineering, 223 Transportation Research Building, University of Kentucky, Lexington, KY 40506-0043, U.S.A.)

July $23-27,1984$

- CHALLENGES IN AFRICAN HYDROLOGY AND WATER RESOURCES (International Symposium), Harare, Zimbabwe. Sponsored by IAH, IAHS, Unesco. Post-symposium tour. Languages: English and French. (Dr. J.C. Rodda, Institute of Hydrology, Wallingford, Oxon., X 10 8BB, U.K.)

July $24-26,1984$

ROCK-OLYMPIC USA (International Symposium, rock engineering), Las Vegas, Nevada, U.S.A. (Dr. Yung Sam Kim, Nevada Institute of Technology, P.O. Box 8894, Campus Station, Reno, NV 89507 , U.S.A.)

July 29 - August 4, 1984

EUROGEOPHYSICS (10th Assembly), Louvain-laNeuve, Belgium. Sponsored by European Geophysical Society. (Prof. A. Berger, institut d'Astronomie et de Geophysique, B-1348 Louvain-laNeuve, Belgium)

July 31 - August 1984

FISSON TRACK DATING (Workshop), Troy, New York, U.S.A. (Donald S. Miller, Department of Geology, Rensselar Polytechnic Institute, Troy, NY 12181)

August 1984

MAPPING OF THE SOIL-WATER BALANCE (Meeting), Budapest, Hungary. (Dr. W.G. Sombroek, ISSS, International Soil Museum, 9 Duivendaal, POB 353, 6700 A.J. Wageningen, The Netherlands)

August $4-14,1984$

* 27th INTERNATIONAL GEOLOGICAL CONGRESS, Moscow, U.S.S.R. (N.A. Bogdanov, Secre tary General, 27 th IGC, Staromonetny per. 22, Moscow 109180 , U.S.S.R.) See detailed list below.

August $6-8,1984$ STABILITY IN UNDERGROUND MINING (2nd International Conference), Lexington, Kentucky, U.S.A. (Department of Mining Engineering, University of Kentucky, Lexington, KY 40506, U.S.A.)

August $6-9,1984$

FINE-GRAINED SEDIMENT: ORIGINS, TRANS PORT AND DISTRIBUTION (SEPM Research Conference), San Jose, California. (D.S. Gorsline, Dept. Geological Sciences, University of Southern California, Los Angeles, CA 90089-0741, U.S.A.)

August $9-18,1984$

CRYSTALLOGRAPHY (13th General Assembly), Hamburg, F.R.G. (E.E. Snider, American Crystal- lographic Association, 335 East 45th Street, New York, NY 10017 , U.S.A.)

August $10-14,1984$

AUSTRALASIAN INSTITUTE OF MINING AND METALLURGY (Annual Conference), Darwin, Northern Territories, Australia. (A.P. Bravo, Aus. IMM Darwin Branch, P.O. Box 1510, Darwin, NT 5794, Australia)

August $13-17,1984$

- CHEMICAL OCEANOGRAPHY (Gordon Research Conference), Meriden, New Hampshire. (A.M. Cruickshank, Pastore Chernical Laboratory, Univ. of Rhode Island, Kingston, RI 02281, U.S.A.)

August $18-24,1984$

INTERNATIONAL PALEOBOTANICAL CONFER ENCE, Edmonton, Alberta, Canada. Sponsored by the International Organization of Paleobotany, to take place before the 6th International Palynological Conference. Field excursions. (Dr. Ruth A. Stockey, Dept. of Botany, The University of Alberta, Edmonton, Alberta, Canada T6G 2E9)

August $18-28,1984$

- RELATIONSHIP BETWEEN GLACIAL TERRAIN AND GLACIAL SEDIMENT FACIES (Annual Meeting INQUA Commission on Genesis and Lithology of Quaternary Deposits), Lethbridge, Alberta, Canada. (Dr. Mark M. Fenton, Aberta Geological Survey, 4445 Calgary Trail South, Edmonton, Canada T6G 5R7)

August 19 - September 2, 1984

- FIELD SYMPOSIUM ON THE CALEDONIDES OF THE BRITISH ISLES (NATO Institute), U.K. (A.L. Harris, Dept. of Geology, University of Liverpool, Box 147, Liverpool, L69 3BX, U.K.)

August $20-24,1984$

ORGANIC GEOCHEMISTRY (Gordon Research Conference), Plymouth, New Hampshire, (R.H. Reitsma, Marathon Oil Co., Box 269, Littleton, CO 80160 , U.S.A.)

August $20-24,1984$

- ORIGIN OF LIFE (Gordon Research Conference), New London, N.H., (A.M. Cruickshank, Pastore Chemical Laboratory, Univ. of Rhode Island, Kingston, RI 02281, U.S.A.))

August 24 - 30, 1984

- 6TH INTERNATIONAL PALYNOLOGICAL CONFERENCE, Calgary, Alberta, Canada. Sponsored by ICP, CSPG, the University of Calgary, and Arctic Institute. Pre- and post-conference excursions. (L. Kokoski, Conference Office, Faculty of Continuing Education, Education Tower Room 102, Calgary, Alberta, Canada T2N IN4)

August $26-31,1984$

- THE ROLE OF AUSTRALIA IN STRENGTHENING THE GEOSCIENCES IN DEVELOPING COUNTRIES (Serninar), Canberra, Australia. Sponsored by AGID and ILP. (M.B. Katz, School of Applied Geology, University of New South Wales, Box 1, Kensington, N.S.W. 2033, Australia)

August $26-31,1984$

GEOLOGICAL CONVENTION AND GEOSCIENTIFIC EXHIBITION (7th Australian), North Ryde, NSW. (Secretary 7AGC, P.O. Box 383, North Ryde, NSW 2113, Australia)

August $27-31,1984$

SYMPOSIUM ON ICE (7th IAHR), Hamburg, F.R.G. (J. Schwarz, Ice Engineering Div., Hamburgische Schiffbau-Versuchanstalt GmbH., P.O. Box 600929,2000 Hamburg, F.R.G.)

August 27 - 31, 1984

25TH INTERNATIONAL GEOGRAPHICAL CONGRESS (and Meeting of the 16th General Assembly of the International Geographical Union), Paris, France. Languages: French, English, German, and Italian. (Comité d'Organisation du Congrès International de Géographie, 19 rue Isidore-Pierre, 14000 Caen, France)

August $27-31,1984$

WATER MOVEMENT IN HEAVY CLAY SOILS (In- 


\section{Coming Events}

ternational Symposium), Wageningen, Netherlands. (Dr. W.G. Sombroek, ISSS, Internationa Soil Museum, 9 Duivendaal, POB 353, 6700 A.J. Wageningen, The Netherlands)

August $28-31,1984$

WATER AND WASTE ENGINEERING FOR DEVELOPING COUNTRIES. (Conference), Singapore. (John Pickford, WEDC Group Leader, University of Technology, Loughborough, Leics. LEII 3TU, U.K.)

September 1984

- MINING TECHNIQUES FOR ALLUVIAL TIN DEPOSITS (International Seminar), Ipoh, Malaysia. (The Director, SEATRAD Centre, Tiger Lane, Ipoh, Malaysia)

September 1984

- TYRRHENIAN OF ALGERIA (International Field Meeting), Algeria. Organised by INQUA. (Centre de Recherches Anthropologiques, Préhistoriques et Ethonographiques (CRAPE), Institut des Sciences Humaines, Le Bardo, 3, rue Franklin D. Roosevelt, Alger, Algeria)

September 1984

* INTERGLACIAL MARINE DEPOSITS, PAST AND PRESENT (Symposium), Schleswig-Holstein, F.R.G. Sponsored by INQUA. (Dr. H. Streiff, Niedersächs, Landesamt fÜr Bodenforschung, Postfach 5101 52, D-3000 Hannover 51, F.R.G.)

September 2 - 7, 1984

SNOW AND ICE PROCESSES AT THE EARTH'S SURFACE (Symposium), Sapporo, Japan. (Mrs. H. Richardson, Secretary General, International Glaciological Society, Lensfield Road, Cambridge CB2 1ER, U.K.)

September $3-5,1984$

- DYNAMIC SOIL STRUCTURE INTERACTION (International Symposium), Minneapolis, U.S.A. (International Symposium on Dynamic Soil-Struc ture Interaction, Department of Civil and Minera. Engineering, 500 Pillsbury Drive SE, University of Minnesota, Minneapolis, MN 55455, U.S.A.)

September 3 - 6, 1984

- JURASSIC STRATIGRAPHY (IUGS Subcommission International Symposium), Er langen, F.R.G. (Prof Dr. A. Zeiss, Paläontol. Inst. Univ. Loewenichstrasse 28, D-8520 Erlangen, F.R.G.)

September $3-6,1984$ DESIGN AND PERFORMANCE OF UNDERGROUND EXCAVATIONS (International Symposium), London, U.K. (Miss Y. Brooks, ISRM 1984 Symposium Conference Office, Institution of Civil Engineers, Great George Street, Westminster, London SW 1 JAA, U.K.)

September 3-8, 1984

- EVOLUTION OF THE CALEDONIAN-APPALACHIAN OROGEN (Final Symposium of IGCP Project 27), Glasgow, Scotland. (A.L. Harris, The University of Liverpool, Jane Herdman Laboratories of Geology, Brownlow Street, P.O. Box 147, Liverpool L69 3BX, U.K.)

September $6-9,1984$

- MEDITERRANEAN NEOGENE, MARINE MEGAFAUNAL PALAEOENVIRONMENTS, AND BIOSTRATIGRAPHY (Interim Colloquium, RCMN), Athens, Greece. (Prof. E. Georgiades-Dikeoulia, Laboratory of Stratigraphy and Palaeontology, Athens University, Panepistimiopolis, Post Office Zografou, Athens 15701, Greece)

September 6 - 11, 1984

MESOZOIC TERRESTRIAL ECOSYSTEMS (Symposium), Stuttgart - Tübingen, F.R.G. Language: English. (Dr. Frank Westphal, Institut und Museum fur Geologie und Paläontologie, Sigwartstrasse 10, D-7400 Tübingen 1, F.R.G.)

September $8-13,1984$

- EUROPEAN ASSOCIATION OF SCIENCE EDITORS and COUNCIL OF BIOLOGY EDITORS (Conference), Cambridge, U.K. (EASE Secretary/Treasurer, Nancy Morris, P.O. Box 33, Farnham, Surrey GU10 3JX, U.K.)
September $10-12,1984$

- OCEANS 84 (Conference), Washington, D.C. Oceans 84 Technical Program Committee, 1730 M St. NW, Suite 412, Washington, D.C. 20036 , U.S.A.)

September $10-14,1984$

TITANIUM (5th International Conference), Munich, F.R.G. (Deutsche Gesellschaft fur Metallkunde EV, Adenauerallee 21, D-6370 Oberursel 1, F.R.G.)

September $11-12,1984$

HABITAT OF PALAEOZOIC GAS IN NW EUROPE (Meeting), London, U.K. Geological SocietyPetroleum Group. (Geological Society, Burlington House, Piccadilly, London WIV OJU, U.K.)

September $11-15,1984$

- GEOLOGY OF BOLIVIA (2nd Congress), Cocha bamba, Bolivia. Language: Spanish. (Secretary General, II Geological Congress of Bolivia, Casilla 183, Cochabamba, Bolivia)

September 12 - 14, 1984

- DEGRADATION, RETENTION, AND DISPERSION OF POLLUTANTS IN GROUNDWATER (Seminar), Copenhagen, Denmark. (Erik Arvin, Department of Environmental Engineering, Building $115 \mathrm{C}$, Technical University of Denmark, DK-2800 Lyngby, Denmark)

September 12 - 14, 1984

ALKALINE IGNEOUS ROCKS (Geological Society of London Symposium), Edinburgh, Scotland. (J.G. Fitton, Grant Institute of Geology, West Mains Road, Edinburgh EH9 3JW, Scotland, U.K.)

September 13 - 19, 1984

DYNAMICAL AND CHRONOLOGICAL RELATIONS BETWEEN GLACIAL AND PERIGLACIAL DEPOSITS (Annual Meeting INQUA Subcommission on European Quaternary Stratigraphy), Besançon, France. (Dr. Michel Campy, Labo de Géologie Historique, Institut des Sciences Naturelles, Place Leclerc, F-25030 Besançon, France)

September $14-16,1984$

GEOLOGY AND GENESIS OF MINERAL DEPOS. ITS IN IRELAND (International Conference), Dublin, Ireland. (J. Ashton, Tara Mines Geology Dept., Knockumber, Co. Meath, Ir eland)

September $16-22,1984$

* LANDSLIDES (4th International Symposium), Toronto, Canada. Sponsored in part by IAEG. (Mr. J.L. Seychuk, Chairman, Organizing Committee, ISL/84, P.O. Box 370, Station A, Rexdale, Ont., Canada M9W 5L 3)

September 17 - 20, 1984

AQUATECH '84. (12th international Congress), Amsterdam, The Netherlands. Sponsored by the international Association for Water Pollution Research. (IAWPR, Alliance House, 29/30 High Holborn, London WC1V 6BA, U.K.)

September 20 - 25, 1984

RECENT ADVANCES IN PETROLEUM EXPLORATION AND DEVELOPMENT (Meeting), Beijing, P.R. China. Co-sponsored by CPEMRC and Chinese Petroleum Geology Society, (R.J. Foster, BHP Petroleum, G.P.O. Box 1911R, Melbourne, 3001 Australia)

September $23-28,1984$

- TRANSPORT PROCESSES IN FRACTURE ROCK (Penrose Conference), Park City, Utah. (L.J. Smith, Dept. of Geological Sciences, Univ. of British Colombia, Vancouver, B.C., Canada V6T 2B4)

September $24-28,1984$

- ASSESSMENT OF SOIL SURFACE SEALING AND CRUSTING (International Symposium), Ghent, Belgium. Language: English. (Organizing Committee, International Conference, Department of Soil Physics, State University of Ghent, Coupure Links 653, 9000 Ghent, Belgium)
September $24-28,1984$

ICSU (20th Genera! Assembly), Ottawa, Canada. K. Charbonneau, Conference Office, National Research Council, Ottawa, Canada KIA OR6)

September 25, 1984

- Global CHANGE (ICSU Symposium), Ottawa, Canada. (K. Charbonneau, Conference Office, National Research Council, Ottawa, Canada K1A OR6)

September 25 - 27, 1984

- FOSSIL ARTHROPODS AS LIVING ORGANISMS (International Symposium), Edinburgh, U.K. (Royal Society of Edinburgh, 22-24, George St., Edinburgh EH2 2PQ, U.K.)

September $26-28,1984$

SEDIMENTATION IN THE AFRICAN RIFT SYSTEM (Geological Society Meeting), London, U.K. (L.E. Frostick, Dept. of Geology, Birkbeck College, 7/15 Gresse Street, London W IP IPA, U.K.)

September 30 - October 6, 1984

* late QuATERnARY SEA-LEVEl CHANGES AND COASTAL EVOLUTION (International Symposium and Field Meeting), Argentina and Chile. IGCP-200 and INQUA Commission on Quaternary Shorelines. (Dr. Enrique Schnack, International Sea-level Symposium, Casilla 722, Correo Central, 7600 Mar del Plata, Argentina)

October 1 - 5, 1984

- SCIENTIFIC COMMITTEE ON ANTARCTIC RESEARCH (18th Meeting), Bremerhaven, F.R.G. (G. Hemmen, Scott Polar Research Institute, Lensfield Road, Cambridge, U.K. CB2 IER)

October 1 - 5, 1984

EUROPEAN SEISMOLOGICAL COMMISSION (Assembly), Moscow, U.S.S.R. (Prof. A.V. Nikolayev, Institute of Physics of the Earth, Academy of Sciences of U.S.S.R., Bolshaya Gruzinskaya, 10, Moscow 123 242, U.S.S.R.)

October 1 - 5, 1984 REMOTE SENSING OF ENVIRONMENT (18th international Symposium), Paris, France. (Environmental Research Institute of Michigan, P.O. Box 8618, Ann Arbor, MI 48107, U.S.A.)

October 1 - 6, 1984

CENTRAL ANDEAN TECTONICS (Symposium), La Paz, Bolivia. (Secretaria, Commission National de Estudios Geofisicos, Casilla 5939, La Paz, Bolivia)

October 5, 1984

WATER RESOURCES PLANNING AND MANAGEMENT (International Conference), Athens, Greece. (Prof. A. Aureli, Via Cimarosa 10,95124 Catania, Italy)

October 7 - 12,1984

- A BUENOS AIRES COASTAL Plain - NORTH PATAGONIA COAST (Field Meeting), Mar de Plata, Argentina. INQUA Shorelines Commission. (Dr. Enrique Schnack, Casilla 722, Correo Central, RA - 7600 Mar del Plata, Argentina)

October 8 - 10, 1984

- ASSOCIATION OF EARTH SCIENCE EDITORS (Annual Meeting), Portland, Oregon, U.S.A. (Beverly Vogt, Oregon Department of Geology, 1005 State Office Building, Portland, OR 97201, U.S.A.)

October $8-12,1984$

BIOGEOCHEMICAL CYCLING OF 5 AND $N$ IN REMOTE AREAS (NATO Workshop), St. Georges, Bermuda. (J.N. Galloway, Environmental Studies Dept., University of Virginia, Char lottesville, VA 220903, U.S.A.)

October $9-14,1984$

IN SITU SOIL AND ROCK REINFORCEMENT (International Conference), Paris, France. (Conference Director, ENPC/DFCAI, 52, rue Madame, 75006 Paris, France)

October $13-16,1984$

- ORIGIN OF THE MOON (Topical Conference) Kona, Hawaii. (P. Jones, LPI, 3303 NASA Road One, Houston, TX 77058 , U.S.A.) 


\section{Coming Events ...}

October $14-20,1984$

MINERAL PROCESSING AND EXTRACTIVE METALLURGY. (International Conference), Kunming, P.R. China. (The Secretary, Institution of Mining and Metallurgy, 44 Portland Place, London WIN 4BR, U.K.)

October $15-17,1984$

- SINKHOLES (lst Multidisciplinary Conference) Orlando, Florida, U.S.A. (College of Extended Studies, University of Central Florida, Orlando, FI 32816)

October $15-18,1984$

- LATIN AMERICAN CONGRESS OF PALAEONTOLOGY (3rd Congress), Oaxtepec, Morelos, Mexico. (Dr. Jose C. Guerrero, Universidad Nacional Autonoma de Mexico, Mexico D.F., Mexico)

October $17-19,1984$

AIPG (Annual Meeting), Orlando, Florida, U.S.A. (Bobby J. Timmons, Timmons Associates, P.O Box 50606, Jacksonville Beach, FL 32250, U.S.A.)

October $17-20,1984$

AMERICAN ASSOCIATION OF STRATIGRAPHIC PALYNOLOGISTS (Annual Meeting and Field Trip), Arlington, Virginia, U.S.A. (N.O. Frederiksen, U.S. Geological Survey, M.S. 970, Reston, VA 22092, U.S.A.)

October $21-25,1984$ SOCIETY OF EXPLORATION GEOPHYSICISTS (54th Annual Meeting), Denver, Colorado, U.S.A. (H. Breck, Society of Exploration Geophysicists, P.O. Box 3098, Tulsa, OK 74101 , U.S.A.)

October $24-26,1984$

- NATURE OF THE LOWER CONTINENTAL CRUST (Join t Meeting Geological Society of London with 3rd Alfred Wegener Conference), London. Cosponsored by ILP. (Prof. J.B. Dawson, Department of Geology, The University, Sheffield, SI 3JD, England, U.K.)

October 25 - November 5, 1984

- GEOLOGY OF TIN DEPOSITS (International Symposium), Nanning City, Guangxi Zhuang Autonomous Region, P.R. China. (Mr. Zhang Sihui, Chinese Academy of Geological Sciences, Baiwanzhuang Road 26, Fuchengmenwai, Beijing, People's Republic of China)

October 29 - November 2, 1984

INTERNATIONAL WATER SUPPLY ASSOCIATION (15th International Congress), Tunis, Tunisia. (R. Clark, International Water Supply Association, 1 Queen Anne's Gate, London SW1H 9BT, U.K.)

October 31 - November 7, 1984

- SEISMOLOGY AND PHYSICS OF THE EARTH'S INTERIOR (Regional Assembly of the international Association), Hyderabad, India. Plus short course for developing countries on inversion of geoscience data. Co-sponsored in part by ILP. Corganising Committee, IASPEI Regional Assembly, National Geophysical Research Institute, Hyderabad 500007 , India)

November/December 1984 LAND EVALUATION FOR SOIL EROSION HAZARD ASSESSMENT (Meeting), Enschede, Netherlands. (Dr. W.G. Sombroek, ISSS, International Soil Museum, 9 Duivendaal, POB 353, 6700 A.J. Wageningen, The Netherlands)

November 5-8, 1984

GEOLOGICAL SOCIETY OF AMERICA (Annual Meeting), Reno, Nevada, U.S.A. (S.S. Beggs, Geological Society of America, P.O. Box 9140, 3300 Penrose Place, Boulder, CO 80301 , U.S.A.)

November 5 - 9, 1984 ARGENTINE GEOLOGICAL CONGRESS (9th), Bariloche, Argentina. Field trips. Languages: Spanish, English, and French. (IX Congreso Geológico Argentino, Maipú 645 Piso 1, 1006 Buenos Aires, Argentina)
November $6-7,1984$

NORTH ATLANTIC PALAEOCEANOGRAPHY (Marine Studies Group Meeting), London. (Geological Society, Burlington House, Piccadilly, London W 1, U.K.)

November $12-17,1984$

WATER FOR SOUTH AFRICA (Exposition and Conference), Johannesburg, South Africa. Sponsored by National Water Well Association (U.S.) and Borehole Water Association (S.A.). (Pat Alcorn, NWWA, 500 W. Wilson Bridge Road, Wor thington, Ohio 43085 , U.S.A.)

November 12 - 30, 1984

RURAL HYDROGEOLOGY AND HYDRAULICS IN FISSURED BASEMENT ZONES (Workshop), Roorkee, India. (Prof. B.B.S. Shinghal, Department of Earth Sciences, University of Roorkee, Roorkee 247667 , India)

November 13 - 15, 1984

OPHIOLITES TROUGH TIME (Conference), Nancy, France. (J. Desmons, University de Nancy I, Lab. de Petrologie, B.P. 239, F-54506 Vandoeuvre-lesNancy Cedex, France)

November $15-17,1984$

* MINERAL POLICY FOR SMALL-SCALE MINING (Workshop), New Delhi, India. Cosponsored by AGID in conjunction with World Mining Congress. (Co-ordinator, Regional Mineral Resources Development Centre, P.O. Box 19, Bandung, Indonesia)

November 19-23, 1984

12th WORLD MINING CONGRESS, New Delhi, India. (Organizing Committe, Institute of Engineers, 8 Gokhale Road, Calcut ta 700 020, India)

December 1984

- ClIMAT - MILIEU - HOMME (Colloquium), Dakar, Senegal. Co-sponsored in part by INQUA and Unesco. (Dr. E.S. Diop, University of Dakar, Department of Geography, Dakar-Fann, Senegal)

December 2 - 5, 1984

FUTURE PETROLEUM PROVINCES OF THE WORLD (AAPG W.E. Prat t Memorial Conference), Phoenix, Ariz., U.S.A. (AAPG, P.O. Box 979 , Tulsa, OK 74101 , U.S.A.)

December 2 - 6,1984

SOCIETY OF EXPLORATION GEOPHYSICISTS (54th Annual Meeting), Atlanta, Georgia, U.S.A. (3. Hyden, SEG, Box 3098, Tulsa, OK 74101, U.S.A.)

\section{5}

January 1985

ACID-SULPHATE SOILS (Meeting), Dakar, Senegal. (Dr. W.G. Sombroek, ISSS, International Soil Museum, 9 Duivendaal, $\mathrm{POB} 353,6700$ A.J. Wageningen, The Netherlands)

January 7 - 10,1985

HYDROGEOLOGY OF ROCKS OF LOW PERMEABILITY (17th International Congress of IAH), Tucson, Arizona, U.S.A. (Eugene S. Simpson, Department of Hydrology and Water Resources, College of Earth Sciences, The University of Arizona, Tucson, AZ 85721 , U.S.A.)

February 1985

INTERGOVER NMENTAL OCEANOGRAPHIC COMMISSION ASSEMBLY (13th Session), Paris, France. (Unesco, 7, place de Fontenoy, 75700 Paris, France)

February 11 - 14, 1985

GEOMECHANICS IN TROPICAL LATERITE AND SAPROLITIC SOILS (1st International Conference), Sao Paulo, Brazil. (Dr. W.C. Hachich, Secretary ISTS-BMS, C.P. 7141, 01000 Sao Paulo, SP, Brazil)

February $11-14,1985$

ASIAN MINING '85 (2nd Conference), Manila, Philippines. (Meeting Secretary, The Institution of Mining and Metallurgy, 44 Portland Place, London WIN 4BR, U.K.)
February 27 - March 2, 1985

GEOLOGY OF THE OCEANS (75th Annual meet ing of the Geologische Vereinigung), Kiel, West Germany. Languages: English and German. (M. Sarnthein, Geologisch-Palaeontologisches Institut, Universitaet, Olshausenstrasse, D-2300 Kiel, F.R.G.)

March $11-15,1985$

SE ASIAN GEOTECHNICAL CONFERENCE (8th), Kuala Lumpur, Malaysia. Language: English. (The Hon. Secretary, 8th SEAGC, The Institution of Engineers, Malaysia, P.O. Box 223, Petaling Jaya, Selangor, Malaysia)

March 11 - 15,1985

- TUNNELliNG 85 (4th International Symposium), Brighton, England. (Tunnelling 85, The Secretary, Institution of Mining and Metallurgy, 44 Portland Place, London W IN 4BR, U.K.)

April 1 - 4, 1985

- EUROPEAN UNION OF GEOSCIENCES (Biennia Conference), Strasbourg, France. (Organizing Committee, Department of Earth Sciences, University of Cambridge, Downing Street, Cambridge CB2 3EQ, U.K.)

April $1-5,1985$

NUMERICAL METHODS IN GEOMECHANICS (5th International Conference), Nagoya, Japan. (Prof. T. Kawamoto, Department of Civil \& Geotechnical Engineering, Nagoya University, Chikusa, Nagoya 464, Japan)

April $9-12,1985$

EVOLUTION OF THE EUROPEAN LITHOSPHERE (MEGS 4: Meeting of European Geological Societies), Edinburgh, U.K. (Dr. S.K. Monro, Institute of Geological Sciences, Murchison House, West Mains Road, Edinburgh EH9 3LA, Scotland, U.K.)

April 14 - 17, 1985

PROSPECTING IN AREAS OF DESERT TERRAIN (Conference), Rabat, Morocco. (Conference Office, IMM, 44 Portland Place, London WIN 4BR, U.K.)

April 28 - May 1, 1985

- GEOCHEMICAL EXPLORATION (11th International AEG Symposium), Toronto, Canada. (Dr. W.B. Coker, Kidd Creek Mines Ltd., 357 Bay St. Ste. 300, Toronto, Ontario, Canada M5H 1T7)

May $6-17,1985$

- NEOGENE PHOSPHORITES OF THE SE UNITED STATES (International field workshop and seminar, IGCP 156), Greenville, N.C., to Tallahassee, Florida. (W.C. Burnett, Dept. of Oceanography, Florida State University, Tallahassee, FL 32306, U.S.A.)

May $13-17,1985$

TUNGSTEN (3rd International Symposium), Madrid. (Mr. M.R.P. Maby, Secretary, Primary Tungsten Association, 280 Earls Court Road, London SW 5 9AS, U.K.)

May $15-17,1985$

TURBIDITE-HOSTED GOLD DEPOSITS (International Symposium), Fredericton, New Brunswick, Canada. Symposium held with Geological Association of Canada Annual Meeting. (Simon J. Haynes, Nova Scotia Department of Mines and Energy, P.O. Box 1087, 1690 Hollis Street, Halifax, Nova Scotia, Canada B3J 2X1)

May 27 - 31, 1985

AMERICAN GEOPHYSICAL UNION (Spring Meeting), Baltimore, Md. (Meetings, AGU, 2000 Florida Avenue, NW, Washington, DC 20009, U.S.A.)

May 27 - June 1, 1985

CORAL REEF CONGRESS: Reef and Man (5th International), Papeete, Tahiti. (Antenne Museum Ephe, Congrès Récifs Coral liens 1985, B.P. 562, Papeete, Tahiti, French Polynesia)

June $2-9,1985$

INTERNATIONAL MINERAL PROCESSING CONGRESS (15th), Cannes, France. Languages French 


\section{Coming Events}

and English. (International Mineral Processing Congress Secretary, BRGM SGN/Mineralurgie, B.P. 6009-45060 Orléans Cedex, France)

June $9-15,1985$

WATER RESOURCES ( 5 th World Congress), Brussels, Belgium. (Dr. L.W. Debacker, c/o Brussels International Conference Centre, Parc des Expositions, Place de Belgique, B-1020 Brussels, Belgium)

June $16-21,1985$

SEISMICITY AND SEISMIC RISK (3rd Internationa! Symposium), Liblice, Czechoslovakia. (Dr. Z Schenkova, Geophysical Institute, Bocni II, 14131 Prague 4, Czechoslovakia)

July 7 - 19, 1985

KARST WATER RESOURCES (International Symposium), Antalya/Ankara, Turkey. Sponsored by IAHS. (Prof. G. Gunay, Hydrogeological Engineering Department, Hacettepe University, Beytepe, Ankara, Turkey)

July $14-30,1985$

- MAGMATIC SULFIDES IN MAFIC ROCK (IGCP 161 - Field Conference 4), Ketchikan, Alaska to Duluth, Minn. (Gerald K. Czamanske, M.S. 984, U.S. Geological Survey, Menlo Park, CA 94025 , U.S.A.)

July 27 - August 7, 1985

- 4 th INTERNATIONAL PLATINUM SYMPOSIUM, Duluth, Minnesota, to Sudbury, ontario. (A.J. Naldrett, Department of Geology, University of Toronto, Toronto, Ontario, Canada M5S 1A1)

July 28 - August 2, 1985

- 8th INTERNATIONAL CLAY CONFERENCE, Denver, Colorado, U.S.A. Sponsored by AJPEA. (Dr. A.J. Herbillon, Groupe de Physico-Chimie Minérale et de Catalyse, Univ. Catholique de Louvain, Place Croix du Sud 1, B-1348 Louvain-laNeuve, Belgium)

July 29 - August 1, 1985

- OSTRACODA (9th International Symposium), Shizuoka, Japan. (Dr. Tetsuro Hanai, Institute of Geosciences, University of Shizuoka, Shizuoka, 422, Japan)

August 5 - 17, 1985

MAGNETIC ANOMALIES OVER MARGINS OF CONTINENTS AND PLATES (IAGA Symposium), Prague, Czechoslovakia. (W.J. Hinze, Department of Geosciences, Purdue University, West La Fayette, IN 47907, U.S.A.)

August 6 - 10, 1985

SCIENCE AND TECHNOLOGY EDUCATION AND THE QUALITY OF LIFE (International Conference), Bangalore, India. Sponsored by ICSU/CTS (J. Lewis, Physics Department, Malvern College, Malvern, Worcs., U.K.)

August $6-10,1985$

GROUND FREEZING (4th International Symposium), Sapporo, Japan. (ISGF85, Institute of Low Temperature Science, Hokkaido University, Sapporo 060, Japan)

August $11-15,1985$

INTERNATIONAL SOCIETY FOR SOIL MECHANICS AND FOUNDATION ENGINEERING (11th International Conference), San Francisco, California, U.S.A. (K. Hyland, American Society of Civil Engineers, 345 East 47th Street, New York, NY 10017, U.S.A.)

August $19-23,1985$

- SIXTH GONDWANA SYMPOSIUM. Columbus, Ohio, U.S.A. Sponsored by IUGS and Geological Society of America. (D. Elliott, Inst. of Polar Studies, Ohio State University, 103 Mendenhall, 125 South Oval Mall, Columbus, OH 43210 , U.S.A.)

August $19-30,1985$

IASPEI (23rd General Assembly), Tokyo, Japan.

(Prof. R. Sato, c/o Inter Group Corp., Akasaka Yamakatsu Building, 8-5-32 Akasaka, Minato-ku,
Tokyo 107, Japan)

August 24 - September 2, 1985

- a GRAPTOLITES (3rd International Conference, Graptolite Working Group IPA), Helsingor, Denmark. (Dr. M. Bjerreskov, Institute of Historical Geology and Palaeontology, University of Copen-

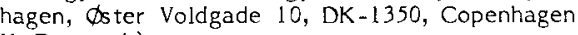
$\mathrm{K}$, Denmark)

August 26 - 29, 1985

GLACIER MAPPING AND SURVEYING (Symposium), Reykjavik, Ic eland. (Mrs. H. Richardson, Secretary General, International Glaciological Society, Lensfield Road, Cambridge CB2 IER, U.K.)

\section{September 1985}

VARIATIONAL METHODS IN GEOSCIENCES (International Symposium), Norman, Oklahoma, U.S.A. (CIMMS, Attn: Y.K. Sasaki, The University of Oklahoma, 815 Jenkins, Norman, Oklahoma 73019 , U.S.A.)

September 1985

ROLE OF ROCK MECHANICS IN MINING (International Symposium), Mexico City, Mexico. (Sociedad Mexicana de Mecanica de Rocas AC, Camino a Santa Teresa 187, Villa Olimpica, MEX14020 Mexico DF, Mexico)

September $2-7,1985$

- FORELAND BASINS (Research Symposium), Fribourg, Switzerland. Sponsored by IAS. (P. Homewood, Institut de Géologie, Université de Fribourg, Pérolles, $\mathrm{CH}-1700$ Fribourg, Switzerland)

September 3 - 5, 1985

EROSION DEBRIS FLOW AND DISASTER PREVENTION (International Symposium), Tsukuba, Japan. (Dr. S. Kobaski, ISEDD 1985, Department of Forestry, Kyoto University, Kyoto 606, Japan)

September 5 - 10, 1985

- CARPATHO-BALKAN GEOLOGICAL ASSOCIATION (13th Congress), Krakow, Poland. (Oddzial Karpacki Insty tutu Geologiczhego, ul. Skrzatow 1, 31-560 Krakow, Poland)

September 8 - 13,1985

* HYDROGEOLOGY IN THE SERVICE OF MAN (18th IAH Congress - International Symposium), Cambridge, U.K. (J. Day, Hydrogeology Unit, Maclean Building, Crowmarsh Gifford, Walling ford, OX10 8BB, U.K.)

September $9-13,1985$

FOSSIL AND LIVING BRACHIOPODS (Meeting), Brest, France. (Congrès Brachiopodes, Univ. Bretagne Occidentale, Laboratoire du Paléozoique - 6, av. Le Gorgen, 29283 Brest Cedex, France)

September $15-20,1985$

BASEMENT TECTONICS (6th International Conference), Santa Fe, New Mexico, U.S.A. Sponsored by Basement Tectonics Association. Pre- and post-conference field trips. (Dr. M. James Aldrich, Mail Stop D461, P.O. Box 1663, Los Alamos National Laboratory, Los Alamos, NM 87545, U.S.A.)

September $15-21,1985$

GEOMORPHOLOGY, RESOURCES, ENVIRONMENT AND THE DEVELOPING WORLD (International Conference), Manchester, U.K. (Prof. Ian Douglas, School of Geography, University of Manchester, Manchester M13 9PL, U.K.)

September 15 - 22, 1985

$\triangle$ REgIONAL COMMITTEE ON MEDITERRANEAN NEOGENE STRATIGRAPHY (8th Congress), Budapest, Hungary. Excursions. Language: English. (Organizing Committee of the 8th Congress of the RCMNS, Hungarian Geological Survey, Népstadion ut 14. H-1442 Budapest, P.O.B. (Pf.) 106, Hungary)

September $16-21,1985$

GEOPHYSICAL AND PETROLOGICAL CONSTRAINTS TO MAGMA GENERATION (IAVCEI Scientific Assembly), Catania, Italy. (G. Frazetta, Istituto Internazionale di Vulcanologia, Viale
Regina Margherita 6,95123 Catania, Italy)

September $19-21,1985$

GEOLOGICAL SOCIETIES OF THE BRITISH ISLES (6th Meeting), Birmingham, U.K. (Prof. P.A. Garrett, Department of Geological Sciences, The University of Birmingham, P.O. Box 363, Edgbaston, Birmingham B15 $2 \mathrm{~T}^{\mathrm{T}}$, U.K.)

September $22-26,1985$

HIGH HEAT PRODUCTION, GRANITES, HYDROTHERMAL CIRCULATION AND ORE GENESIS (Conference), St. Austell, Cornwall, U.K. (IMM, 44 Portland Place, London WIN 4BR, U.K.)

September 22 - 28, 1985

CHEMRAWN IV: CHEMISTRY AND RESOURCES OF THE GLOBAL OCEAN (Meeting), Woods Hole, Mass., U.S.A. (Prof. G. Ourisson, Centre de Neurochimie, Université Louis Pasteur, 5 rue Blaise Pascal, F-67084 Strasbourg, France)

October $5-12,1985$

- DISPOSAL OF HAZARDOUS CHEMICAL DEPOSITS (IAEG International Symposium), Winston Salem, N.C., U.S.A. (Dr. M. Primel, L.C.P.C., 58, bould. Lefebvre, 75732 Paris Cedex 15, France)

October $6-10,1985$

SOCIETY OF EXPLORATION GEOPHYSICISTS (55th Annual Meeting), Washigton, D.C., U.S.A. (Convention Assistant, Society of Exploration Geophysicists, P.O. Box 3098, Tulsa, OK 74101 , U.S.A.)

October $14-18,1985$

COAL RESEARCH ( 7 th International Conference), Pretoria, South Africa. (W.G. Jensen, International Committee for Coal Research, Bte 11, B1150 Bruxelles, Belgium)

October $14-18,1985$

- MATHEMATICAL METHODS IN GEOLOGY (International Symposium), Pribram, Czechoslovakia. (Sekretariat symposia, Hornicka Pribram ve vede a Technice, post. schranka 41, Pribram 26102 , Czechoslovakia)

October $28-31,1985$

GEOLOGICAL SOCIETY OF AMERICA (Annual Meeting), Orlando, Florida, U.S.A. (S.S. Beggs, Geological Society of America, P.O. Box 9140 , 330 Penrose Place, Boulder, CO 80301 , U.S.A.)

October 29 - November 2, 1985

- INDONESIAN MINING (International exhibition) Jakarta, Indonesia. (Overseas Exhibition Services, 11 Manchester Square, London WIM 5AB, U.K.)

November 1985

DESERTIFICATION (International Symposium), Khartoum, Sudan. (Dr. W.G. Sombroek, ISSS, International Soil Museum, 9 Duivendaal, $\mathrm{POB}$ 353, 6700 A.J. Wageningen, The Netherlands)

\section{6}

February 1 - 9, 1986

INTERNATIONAL VOLCANOLOGICAL CONGRESS, Auckland, Hamilton, Rotorua, New Zealand. Sponsored by IAVCEI. (P.E. Baker, Department of Earth Sciences, University of Leeds, Leeds LS2 9JT, U.K.)

May $11-16,1986$

MINING AND METALLURGICAL CONGRESS (13th), Canberra, Australia. (Council of Mining and Metallurgical Institutions, 44 Portland Place, London, W $1 \mathrm{~N}$ 4BR, U.K.)

June $1-5,1986$

* GEOSCIENCE INFORMATION (3rd International Conference) Adelaide, South Australia. (Conference Secretariat $31 \mathrm{CGI}$, c/o Australian Mineral Foundation, Private Bag 97, Glenside, South Australia, 5065, Australia)

June 2 - 5, 1986

DINOSAUR SYSTEMATICS (Symposium) Drumheller, Alberta, Canada. (Kenneth Carpenter, Academy of Natural Sciences, 19th and the Parkway, Philadelphia, PA 19103, U.S.A.) 
mmer 1986

GEOCHRONOLOGY, COSMOCHRONOLOGY AND ISOTOPE GEOLOGY (6th International Conference), Cambridge, U.K. Sponsored by IAVCEI, (P.E. Baker, Department of Earth Sciences, University of Leeds, Leeds LS2 9JT, U.K.)

\section{$13-18,1986$}

INTERNATIONAL MINERALOGICAL ASSOCIATION (General Meeting), Stanford, Calif, U.S.A. (Prof. C.T. Prewitt, Department of Earth and Space Sciences, State University of New York, Stony Brook, NY 11794, U.S.A.)

ugust 1986

IAGOD (7th 5ymposium), Lulea, Sweden. (G) Leech, Geological Survey of Canada, 601 Booth Street, Ottawa, Ontario, Canada K1A OE8)

gust $11-15,1986$

KIMBERLITE (4th International Conference), Perth, Western Australia. (Dr. A.F. Trendall, Geological Survey of Western Australia, 66 Adelaide Terrace, Perth, W.A., Australia)

gust $13-20,1986$

SOIL SCIENCE (13th ISSS International Congress), Hamburg, F.R.G. (Prof. Dr. K.H. Hartge, Inst. für Bodenkunde, Univ. Hannover, Herren-häserstrasse 2, D-3000 Hannover 21, F.R.G.)

ugust $25-29,1986$

IAS SEDIMENTOLOGICAL CONGRESS ( 12 th international), Canberra, Australia. (Dr. K.A.W.
Crook, Department of Geology, Australian National University, P.O. Box 5, Canberra, A.C.T., 2600 Australia)

September 7 - 12, 1986

- REMOTE SENSING IN GLACIOLOGY (Symposium), Cambridge, England. (Secretary General, International Glaciological Society, Lensfield Road, Cambridge CB2 lER, England, U.K.)

September $8-12,1986$

- $\triangle$ SECOND INTERNATIONAL CONFERENCE ON PALEOCEANOGRAPHY, Woods Hole, U.S.A. (W.A. Berggren, Woods Hole Oceanographic Institute, Wood's Hole, MA 02543, U.S.A.)

October $20-26,1986$

- IAEG (5th Congress), Buenos Aires, Argentina. (Dr. M. Primel, L.C.P.C., 58, boulevard Lefebvre, 75732 Paris Cedex 15, France)

November $2-6,1986$

SOCIETY OF EXPLORATION GEOPHYSICISTS (56th Annual Meeting), Houston, Texas, U.S.A. (Convention Assistant, Society of Exploration Geophysicists, P.O. Box 3098, Tulsa, OK 74101 , U.S.A.)

November $10-13,1986$ GEOLOGICAL SOCIETY OF AMERICA (Annual Meeting), San Antonio, Texas, U.S.A. (S.S. Beggs, Geological Society of America, P.O. Box 9140,3300 Penrose Place, Boulder, Co 80301 , U.S.A.)
1987

July 31 - August 9,1987

- INQUA (12th Congress), Ottawa, Ontario, Canada. (Dr. Alan V. Morgan, Department of Earth Sciences, University of Waterloo, Waterloo, Ontario, Canada N2L 3G I)

August $9-22,1987$

IUGG (XIX General Assembly), Vancouver, Canada (R.D. Russell, Dept. Geophysics and Astronomy, University of British Columbia, Vancouver, B.C., Canada V6T (W 5 )

September 1987

INTERNATIONAL SOCIETY FOR ROCK MECHANICS (6th International Congress), Montreal, Canada. (Prof. B. Ladanyi, Dept. Civil Engineering, Ecole Polytechnique, Box 6079, Stn.A, Montréal, Canada H3C 3A7)

Wish to announce meetings of international significance? Send brochure or details to Editor, EPISODES, Room 177, 601 Booth Street, Ottawa, Canada $K 1 A O E 8$, at least four manths in advance.
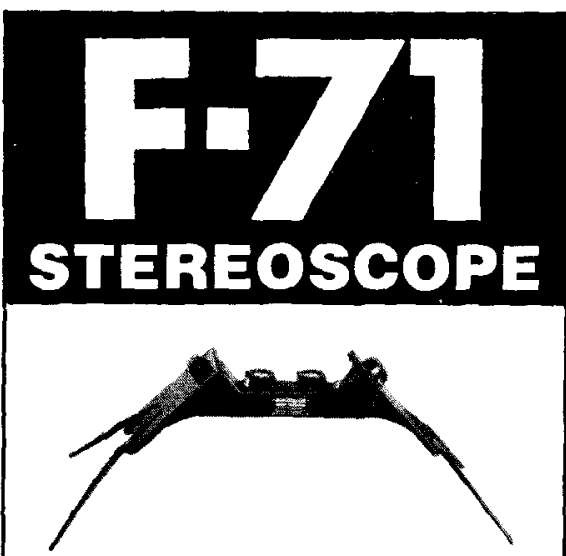

The AGE F-71 Stereoscope is an instrument of fine quality at a low price unmatched in the industry. Its optical system consists of two high-grade, first-surface, vapor-coated mirrors, two $45^{\circ}$ prisms, free of color fringe and two precision-ground diopter lenses. It is supplied with case. An accessory $4 X$ binocular attachment, containing outstanding optics, is available for examining a magnified image. A Parallax Bar, Type 122GE with Starrett precision micrometer, accurate to within $.01 \mathrm{~mm}$, is obtainable. Sold separately or as a package, these precision instruments are the best value on the market today. American made. Write for prices.

Copyright 1979 Alan Gordon Enterprises, Inc.

atan gordon enlertrisos inc. 5362 Cahuenga Blvd., North Hollywood, CA 9160

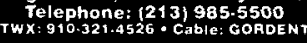

\section{LIST OF ABBREVIATIONS}

$\begin{array}{ll}\text { AGU } & \text { American Geophysical Union } \\ \text { ASCOPE } & \text { ASEAN Council on Petroleum } \\ \text { BRGM } & \text { Bureau de Recherches Géologiques et Minières } \\ \text { CCOP } & \text { ESCAP Committee for Co-ordination of Joint Prospecting } \\ \text { CGMW } & \text { Cor Mineral Resources in Asian Offshore Areas } \\ \text { CEPHPT } & \text { Commission for the Geological Map of the World } \\ & \text { and Temperatures } \\ \text { CIFEG } & \text { Centre International pour la Formation et les Echanges } \\ & \text { Géologiques } \\ \text { CODATA } & \text { ICSU Committee on Data for Science and Technology } \\ \text { COGEODATA IUGS Committee on Storage, Automatic Processing } \\ & \text { and Retrieval of Geological Data } \\ \text { COSPAR } & \text { ICSU Committee on Space Research } \\ \text { CRNS } & \text { Centre Nacional de la Recherche Scientifique } \\ \text { IAEA } & \text { Internationa! Atomic Energy Agency } \\ \text { IAG } & \text { International Association of Geodesy } \\ \text { IAGC } & \text { International Association of Geochemistry and Cosmo- } \\ \text { IAH } & \text { chemistry } \\ \text { IAHR } & \text { International Association of Hydrogeologists } \\ \text { IAHS } & \text { International Association for Hydraulic Research } \\ \text { IASPEI } & \text { International Association of Hydrological Sciences } \\ & \text { International Association of Seismology and Physics } \\ \text { ICS } & \text { of the Earth's Interior } \\ \text { IGCP } & \text { International Commission on Stratigraphy } \\ \text { ICSU } & \text { International Geological Correlation Programme } \\ \text { IGC } & \text { International Council of Scientific Unions } \\ \text { ILP } & \text { International Geological Congress } \\ \text { IMA } & \text { International Lithosphere Program } \\ \text { IMM } & \text { International Mineralogical Association } \\ \text { INHIGE } & \text { Institute of Mining and Metallurgy } \\ \text { International Committee on the History of Geological } \\ \text { IOC } & \text { Sciences } \\ \text { ISSS } & \text { International Union for Quaternary Research } \\ \text { IUGG } & \text { International Oceanographic Commission } \\ \text { SEG } & \text { International Union of Geodesy and Geophysics } \\ \text { SEPM } & \text { Society of Economic Geologists } \\ & \text { Society of Economic Palaeontologists and Mineralogists } \\ & \end{array}$

